\title{
Impacto de la accesibilidad carretera en la calidad de vida de las localidades urbanas y suburbanas de Baja California, México
}

Leonel García. Universidad Autónoma de Baja California, Mexicali, México. Alejandro Mungaray-Moctezuma. Universidad Autónoma de Baja California, Mexicali, México.

Julio Calderón. Universidad Autónoma de Baja California, Mexicali, México. Alejandro Sánchez-Atondo. Universidad Autónoma de Baja California, Mexicali, México.

José Gutiérrez-Moreno. Universidad Autónoma de Baja California, Mexicali, México.

RESUMEN | El impacto de la red carretera federal en la calidad de vida de la población urbana y suburbana en las localidades de Baja California, México, se evalúa mediante un análisis de las condiciones de accesibilidad relativa y absoluta que presenta la red, así como de las intervenciones en materia de conservación carretera en una década. Asimismo, se miden los Índices de Marginación Urbana (IMU) a una escala territorial denominada Área Geoestadística Básica (AGEB), en una situación ex ante y ex post. Se observa que la prioridad ha sido intervenir sobre la red carretera primaria, y que en ciertas localidades de los cinco municipios de la entidad se requiere intervenir en la red alimentadora y secundaria. Se concluye que aquellas localidades con mayor accesibilidad a la red carretera son las que presentan los menores niveles de IMU, principalmente aquellas que hacen intersección con una carretera con anchos de sección transversal más amplios.

PALABRAS CLAVE | calidad de vida, centros urbanos, infraestructura urbana.

ABSTRACT | The impact of the federal highway network on the quality of life of the urban and suburban population in the localities of Baja California in Mexico is evaluated through an analysis of relative and absolute accessibility conditions presented by the network, as well as interventions in road conservation in a decade of study. Likewise, the Urban Margin Indexes (UMI) is measured at a territorial scale called the Basic Geostatistics Area (BGEA), in an ex-ante and ex-post situation. It is observed that the priority has been to intervene on the primary road network and that in certain localities of the five municipalities of the entity it is necessary to intervene in the feeder and secondary network. It is concluded that those localities with greater accessibility within the road network are those with the lowest levels of UMI, mainly those that intersect with a road with wider widths of cross-section.

KEYwORDs | quality of life, urban centre, urban infrastructure. 


\section{Introducción}

El objetivo de este estudio es determinar el nivel de incidencia entre las intervenciones que mejoran las condiciones técnicas de una red carretera, y los cambios en la calidad de vida de la población residente en localidades interconectadas por esa misma red. Se propone una metodología que asocia aspectos de la ciencia social con otros de la ingeniería de vías terrestres, lo que permite alcanzar conclusiones útiles en el ámbito aplicado de la planeación urbana y regional, como también en la administración de los recursos públicos designados para la adecuación de la infraestructura de transporte. El punto de partida de esta investigación es que aquellas localidades urbanas o suburbanas con niveles de marginación urbana mayores son las que presentan los más pobres niveles de accesibilidad carreteros, ya sea por sus condiciones técnicas o por la tipología de la red.

El caso de estudio es la población de 61 localidades urbanas y suburbanas del estado de Baja California, México. El análisis se desarrolla apoyado en la evaluación de las condiciones de accesibilidad relativa y absoluta que presenta su red carretera federal, y la medición de los Índices de Marginación Urbana (IMU) a una escala territorial denominada Área Geoestadística Básica (AGEB) ${ }^{1}$, en el periodo de tiempo 2000-2010. Se realiza una medición cuantitativa y cualitativa de la red para llevar a cabo un análisis de las intervenciones que se han llevado a cabo en la red carretera de la entidad durante el mismo periodo de estudio, tales como trabajos de construcción, rehabilitación y modernización.

Adicional a esta apartado, el artículo está conformado por un análisis del contexto poblacional del caso de estudio y descripción de las características de la red carretera y sus intervenciones en el periodo establecido. Posteriormente, se hace una revisión de las distintas corrientes teóricas que enmarcan las aportaciones de este estudio, relacionadas con el nivel de incidencia de las intervenciones en infraestructura carretera medidas a través de la accesibilidad territorial y en los indicadores que ayudan a determinar la calidad de vida de las personas que residen en una localidad. Se presenta el análisis de accesibilidad desde dos perspectivas: relativa y absoluta. Asimismo, se incluyen los enfoques desde los cuales se analiza la pertinencia de la red, resaltando entre ellos el social, el cual es estudiado a través del IMU. En cuanto a la ruta metodológica que se plantea, esta permite entender la pertinencia social y técnica de las intervenciones carreteras a través de la estimación del IMU por localidad, hasta un nivel territorial urbano comprendido como AGEB. Finalmente, se exponen los resultados de la investigación y las conclusiones.

\section{Contexto poblacional de Baja California}

Baja California es una de las 32 entidades federativas del país. Hace frontera al norte con California y Arizona en los Estados Unidos; al este con Sonora y al sur con Baja

$1 \longdiv { \text { El término AGEB corresponde a un tipo de división territorial urbana que forma parte del Marco } }$ Geoestadístico Nacional (MGN) y es atribuida a una menor escala de dimensionamiento que las demás que lo componen. 
California Sur, del lado mexicano. Cuenta con una superficie de $73.551,5 \mathrm{~km}^{2}$, que corresponde al 3,6\% de la superficie total del territorio nacional (tabla 1). La población total pasó de 2.487 .367 habitantes en el año 2000 a 3.155.070 en 2010, con un aumento de más de 660.000 habitantes, que corresponde a un $26,8 \%$. Al interior del estado, la organización administrativa del territorio se suscita en cinco municipios: Mexicali, Tijuana, Ensenada, Tecate y Playas de Rosarito.

Actualmente, el 92\% de la población estatal reside entre las 61 localidades urbanas y suburbanas del estado, y el 8\% restante en áreas rurales (Instituto Nacional de Estadística y Geografía [INEGI], 2010a), considerándose una zona urbana aquella donde habitan más de 2.500 personas y una zona rural aquella que cuenta con una cantidad de población por debajo de esa cantidad (INEGI, 2010b).

TABLa I | Superficie y densidad de población de Baja California en el año 2000 y 2010

\begin{tabular}{|l|c|c|c|c|c|}
\hline \multicolumn{1}{|c|}{ MUNICIPIO } & $\begin{array}{c}\text { SUPERFICIE } \\
\text { (KM2) }\end{array}$ & $\begin{array}{c}\text { POBLACIÓN } \\
\text { AÑo 2000 } \\
\text { (HAB.) }\end{array}$ & $\begin{array}{c}\text { DENSIDAD DE } \\
\text { POBLACIÓN } \\
\text { AÑo 2OOO } \\
\text { (HAB./KM2) }\end{array}$ & $\begin{array}{c}\text { POBLACIÓN } \\
\text { AÑO 20IO } \\
\text { (HAB.) }\end{array}$ & $\begin{array}{c}\text { DENSIDAD DE } \\
\text { POBLACIÓN } \\
\text { AÑO 2OIO } \\
\text { (HAB./KM2) }\end{array}$ \\
\hline Playas de Rosarito & 506,1 & 63.420 & 125,3 & 90.668 & 179,2 \\
\hline Tecate & $2.724,3$ & 77.795 & 28,6 & 101.079 & 37,1 \\
\hline Tijuana & $1.252,5$ & 1.210 .820 & 966,7 & 1.559 .683 & $1.245,3$ \\
\hline Mexicali & $15.813,2$ & 764.602 & 48,4 & 936.826 & 59,2 \\
\hline Ensenada & $53.255,3$ & 370.730 & 7 & 466.814 & 8,8 \\
\hline Total & $73.551,5$ & 2.487 .367 & 33,8 & 3.155 .070 & 42,9 \\
\hline
\end{tabular}

FUENTE ELABORACIÓN CON BASE EN DATOS DE INEGI 2000, 20IOA

\section{Descripción de la red carretera federal de Baja California}

La red carretera federal estatal se compone principalmente de tres subsectores carreteros: red primaria, red alimentadora y/o secundaria y red rural. En 2010, alcanzaba los 11.129 kilómetros, con 372 kilómetros de carreteras de cuatro carriles; 2.402 kilómetros de carreteras de dos carriles; 4.092 kilómetros de caminos revestidos; 3.092 kilómetros de brechas y 357 kilómetros de terracerías (figura 1) (MungarayMoctezuma \& Luque, 2013). Las carreteras alimentadoras y secundarias alcanzaban un total de 902 kilómetros y los caminos rurales se extienden en 4.182 kilómetros; la red primaria, con un total de 1.705 kilómetros, está conformada por las carreteras federales N. os $1,2,3$ y 5 (INEGI, 2011).

Entre los años 2000 y 2010 se realizaron diferentes trabajos de conservación en la red carretera estatal, con recursos administrados por la Secretaría de Comunicaciones y Transportes. Se identificó que existe una relación entre la cantidad de kilómetros intervenidos, la densidad de población y la dimensión territorial de los municipios. Durante este periodo decenal, del total de intervenciones realizadas en la red primaria, la mayor cantidad se concentró en Ensenada, con un 47,2\%, seguido por Mexicali con el 19,3\%, Tecate con 14,1\%, Tijuana con 12,6\% y Playas 
de Rosarito con 6,8\%. Dichas intervenciones fueron clasificadas principalmente en trabajos de construcción, rehabilitación y modernización de carreteras. La distribución anual que muestra la figura 2 exhibe la preponderancia de esta proporcionalidad, especialmente entre los tres primeros (Ensenada, Mexicali y Tecate), con algunos años donde se pueden percibir porcentajes importantes de inversión en Tijuana (2001 y 2007) y Playas de Rosarito (2005).

\section{FIGURA I | Red carretera en Baja California, 2010}

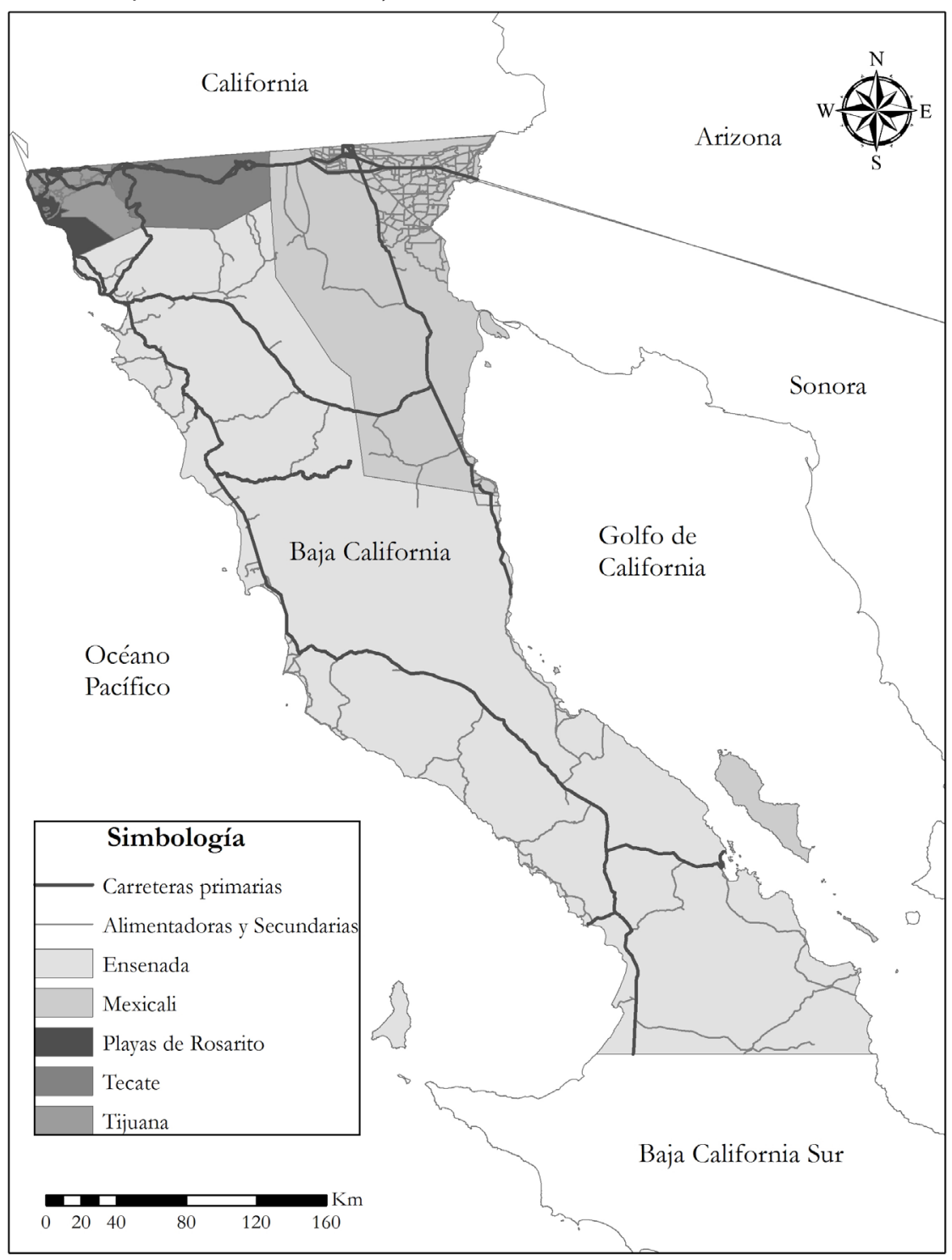

FUENTE ELABORACIÓN PROPIA 
FIGURA 2 | Intervenciones realizadas entre 2000 y 2010 en los municipios de Baja California

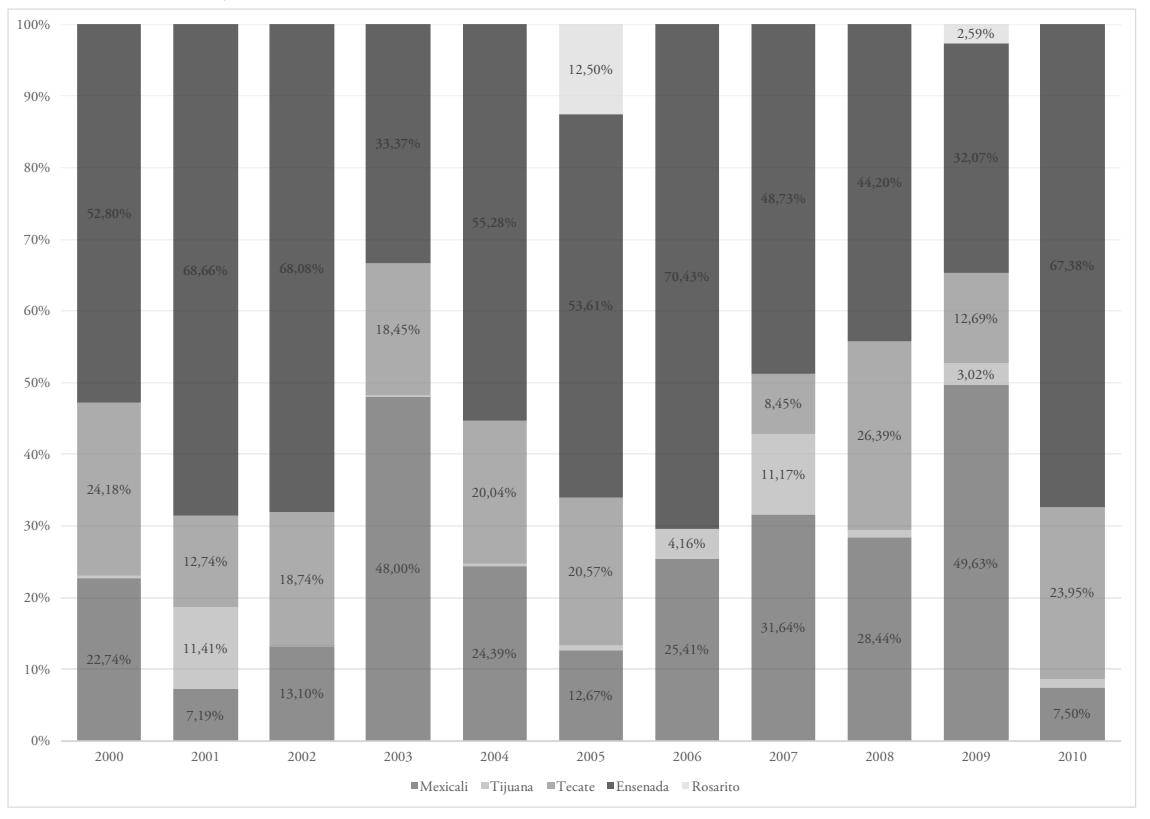

FUENTE ELABORACIÓN PROPIA

\section{Impacto territorial y social de la accesibilidad carretera}

La infraestructura carretera en cualquier comunidad, estado o país representa un factor estratégico para el desarrollo económico y social en su población (Cipolletta, Pérez \& Sánchez, 2010; Vassallo \& Izquierdo, 2010; Zaragoza, 1999). De ella dependen los flujos de mercancías y personas e, indirectamente, el desempeño de los servicios básicos que repercuten en la calidad de la vivienda y, a su vez, en el bienestar de la sociedad (Romero, 2001).

La construcción de carreteras se asocia a procesos de expansión urbana y periférica (Chi, 2010). Su implantación se vuelve un tema primordial en las economías modernas y depende de políticas de gestión e inversión bien planeadas que vean por la desigualdad territorial e impulso del desarrollo social y económico de los países (De Rus Mendoza, 1996; Mungaray-Moctezuma \& García, 2014; ObregónBiosca, 2010). En principio, es porque detonan atracción poblacional, construcción y mejoras en las viviendas, actividades productivas, accesibilidad y ahorros económicos, lo cual debería reflejarse en mejoras en la calidad de vida de la población. Todo ello además de producir efectos benéficos en regiones vecinas (Varela, 2013).

El impacto de la infraestructura carretera primaria en áreas urbanas y suburbanas depende de su trazado, distancias e intersecciones con otros ejes (Coronado \& Garmendia, 2008). Junto con estas, los tramos secundarios y locales forman un tejido fundamental para la mejora de la accesibilidad territorial y atender necesidades de usuarios (Varela, 2013). 
Una gran cantidad de países desarrollados o en desarrollo optan por prácticas de buen gobierno en materia de planeación en infraestructura de transporte, basadas en evaluaciones ex ante y ex post (Bel, 2010).

Farrow y Nelson (2001) consideran la variable accesibilidad como indicador de carencia en políticas de desarrollo en áreas con escaso nivel de urbanización. Repercute en la conectividad poblacional y facilita la movilidad, por lo que se considera en los análisis de competitividad de regiones y países e influye en la toma de decisiones de planeación, construcción y modernización de las carreteras (Geurs \& Van Wee, 2004; International Road Federation [IRF], 2007). Por lo tanto, generar infraestructura que satisfaga las demandas de accesibilidad de la población es una de las tareas más importantes y de mayor responsabilidad para los gobiernos (Lucioni, 2009; Rozas \& Sánchez, 2004).

Loyola (2005) menciona que las cabeceras municipales o zonas más urbanizadas alcanzan mayor accesibilidad que el resto debido a su localización, tamaño poblacional, oferta de servicios y equipamientos. Por otro lado, los sitios con accesibilidad crítica son aquellos que muestran impactos socioeconómicos más severos a consecuencia de la insuficiencia de red y/o conectividad fallida (Taylor, Sekhar \& D'Este, 2006). Por ello, para fines de este estudio, la accesibilidad es vista como un elemento de análisis territorial, principalmente atribuido a la localización de zonas urbanas y suburbanas y a la tipología de la red carretera. Además, se considera la accesibilidad de dichas localidades a ciertos servicios básicos, como lo son educación, salud y vivienda.

Según diversos autores (Gutiérrez Gallego, Mora, Gómez \& Jaraíz, 2010; Gutiérrez \& Monzón, 1993; Loyola, 2005; Loyola \& Albornoz, 2009; Monzón, 1988; y Varela, 2013), en la metodología de análisis de redes carreteras es necesario considerar su tipología y condiciones técnicas para definir el nivel de accesibilidad de la población, considerando dos escenarios de accesibilidad: relativa y absoluta.

Monzón (1988) establece que la accesibilidad relativa considera el grado de conexión de dos puntos situados en el mismo territorio e incorpora el factor distancia en el análisis. Por su parte, para Gutiérrez Gallego et al. (2010), este tipo de accesibilidad muestra con gran nitidez el impacto de las diferentes vías sobre el territorio, debido a que cuantifica el grado de proximidad entre un punto localizado y los principales núcleos urbanos. Por su parte, Loyola (2005) y Nogales, Figueira, Gutiérrez, Pérez y Cortés (2002) plantean que el análisis de la accesibilidad absoluta debe tomar en cuenta variables que actúan como factores de restricción en los usuarios de las carreteras, por lo que se consideran las características técnicas que brinda la red.

El impacto de la infraestructura carretera puede evaluarse desde distintos enfoques. La Secretaría de Comunicaciones y Transportes (sст) (2011) recomienda estudios socioeconómicos, ambientales y técnicos a partir de una metodología que evidencie su pertinencia desde la perspectiva social y legal. En principio, el desarrollo carretero debe vincularse integralmente a la red; es decir, adaptarse a las necesidades sociales y económicas de una región, sin que importe su situación geográfica, y a los efectos que resultan de la relación espacio-tiempo (Obregón-Biosca, 2010). Aunque la infraestructura carretera no impulsa por sí sola el desarrollo regional, la accesibilidad que ofrece a un territorio es un factor clave, principalmente en áreas 
suburbanas con limitadas condiciones de comunicación (Nogués \& Salas, 2007; Bolton, 1990).

La evaluación de carreteras debe representar un instrumento de planeación para potenciar los impactos sociales y económicos que tienen sobre el territorio (Mungaray-Moctezuma \& García, 2014), propiciando el desarrollo y evitando la exclusión social (Cárdenas, 2010; Rodríguez, 2003). Según Aparicio, Jaramillo y San Román (2011), el desarrollo de la infraestructura ha sido una determinante en la superación de la vulnerabilidad de los hogares, sin importar el ámbito geográfico, con beneficios directos por el acceso a bienes, servicios y recursos que ella permite.

En este contexto, el Índice de Marginación Urbana (IMU) es una herramienta útil que permite identificar las condiciones de desarrollo de los habitantes de zonas específicas, medidas por tipos de vivienda y acceso a servicios de salud y de educación (Consejo Nacional de Población [CONAPo], 2012). Esto debido a que la marginación es un fenómeno multidimensional expresado por la distribución de la desigualdad, que refleja la exclusión de la estructura productiva y de diversos grupos sociales a los beneficios del desarrollo (Cárdenas, 2010).

El primer cálculo del IMU en México fue desarrollado en 1990 por CONAPO, y se efectuó en cada entidad federativa y municipio. Su objetivo fue reconocer las carencias de bienes y servicios y la forma como vive la población en determinados espacios territoriales (CONAPO, 2012). Desde entonces este índice ha sido útil para identificar el desarrollo social en áreas urbanas.

Los acercamientos teóricos expuestos en este apartado establecen la pertinencia de realizar un análisis que asocie el comportamiento del IMU de una población urbana y suburbana, con las intervenciones técnicas que se realizan en la infraestructura carretera para mejorar sus condiciones operativas y los niveles de accesibilidad relativa y absoluta que ellas proporcionan.

\section{Metodología: análisis de accesibilidad carretera y su impacto en la calidad de vida en Baja California}

En primera instancia se define la situación actual de la red carretera federal de la entidad, refiriendo los niveles de accesibilidad relativa y absoluta que la red le brinda al territorio para el año 2010. Posteriormente, se determina la situación de la calidad de vida de los habitantes de las localidades que son atendidas por la red en cuestión, esto a través de una clasificación de valores que entrega el cálculo del IMU a partir de seis variables obtenidas de bases de datos oficiales, relacionadas con la calidad de la vivienda y servicios de educación y salud a los cuales se tiene acceso. Para observar el comportamiento de este índice se llevó a cabo el análisis en dos etapas, una ex ante en el año 2000 y otra ex post en el 2010, con el fin de mostrar las variaciones de un año al otro organizadas geoestadísticamente hasta un nivel territorial AGEB.

Otro de los objetivos planteados en esta investigación es determinar si existe coincidencia entre las variaciones del índice, la posición geográfica de la población, la accesibilidad que le otorga la red al territorio y los tipos de intervenciones técnicas que se han llevado a cabo en la propia red durante el periodo de estudio. Esto se hace mediante la comparación de los resultados obtenidos para cada análisis, 
para detectar las localidades que se encuentran en situaciones críticas en términos de accesibilidad territorial por ausencias o deficiencias de infraestructura carretera; y en términos de calidad de vida, en este caso definida por la falta de acceso a servicios básicos como lo son una vivienda digna, servicios médicos adecuados y servicios educativos con instalaciones geográficamente cercanas. Estos dos aspectos se someten a un ejercicio comparativo que los asocia con la georreferenciación de las intervenciones técnicas realizadas en los diversos puntos de red, para determinar si las decisiones tomadas en materia de conservación han sido sensibles a las necesidades básicas de los usuarios que atiende, y han contribuido a disminuir las condiciones de marginación de las localidades de la entidad.

\section{Análisis de accesibilidad territorial brindada por la red carretera}

Estudios como el de Loyola (2005) han concluido que la distancia de recorrido repercute en los niveles de accesibilidad en áreas periféricas. En este sentido, se plantean tres niveles de accesibilidad relativa. El nivel A refiere a localidades directamente asociadas a un trazado primario; el nivel B, a localidades comunicadas a través de un ramal alimentador; y el nivel C, a localidades indirectamente comunicadas a través de la red secundaria o un segundo ramal. Por su parte, la accesibilidad absoluta se determina con variables que manifiestan cierta restricción en los flujos de transporte; específicamente, el tipo de pavimento, ancho de sección transversal y velocidad de operación. Las dos últimas han sido utilizadas por Gutiérrez Gallego et al. (2010) en un análisis de accesibilidad hacia zonas urbanas, resaltando los beneficios atribuidos por el nivel de interconexión que permiten carreteras con diferentes diseńos geométricos y velocidades de operación.

Para representar mejor los datos de accesibilidad, se genera una matriz que incorpora los niveles relativos de cada localidad y las características técnicas predominantes de las carreteras que las conectan, las cuales se obtienen utilizando la herramienta de software del Sistema de Información Geográfica (sIG) ArcGis 10.1.

\section{Análisis de la calidad de vida de la población atendida por la red carretera}

La evaluación de los niveles de calidad de vida de la población se obtiene con el cálculo del IMU en un contexto ex ante (2000) y ex post (2010). Con ello, es posible identificar las tendencias o cambios socioeconómicos de la población de forma georreferenciada, incluso después de estratificar el territorio. Bistrain (2010) resalta la importancia de estratificar el territorio a la menor dimensión posible, con el objeto de obtener resultados más precisos en materia de comportamiento social. En este sentido, es posible calcular el IMU por AGEB (CONAPO, 2012), lo que permite definir con mayor certeza su comportamiento en áreas específicas del territorio.

Los indicadores utilizados para calcular el IMU se relacionan con tres dimensiones: educación, salud y vivienda (CONAPO, 2010). La primera de ellas incorpora un indicador de porcentaje de población sin escuela y/o con primaria incompleta; la segunda, un indicador de porcentaje de población sin derecho a servicios de salud; y la tercera, cuatro indicadores: porcentaje de viviendas particulares habitadas con piso de tierra, porcentaje de viviendas particulares habitadas que no disponen de energía 
eléctrica, porcentaje de viviendas particulares habitadas que no disponen de agua potable, y porcentaje de viviendas particulares habitadas que no disponen de drenaje.

La información que se requiere para obtener el índice por AGEB en los ańos 2000 y 2010 es extraída de las bases de datos de INEGI, específicamente de los Censos de Población y Vivienda. Para conocer la situación educativa se considera: 1) población de 15 años o más, sin escuela o primaria incompleta, para el acceso a servicios de salud; 2) población sin derecho a servicios de salud, para identificar la situación de vivienda; 3) vivienda con piso de tierra; 4) vivienda sin disposición de energía eléctrica; 5) viviendas sin disposición de drenaje y 6) vivienda sin disposición de agua potable.

Para determinar el nivel del IMU de cada AGEB, se considera que el nivel de marginación es medio-alto si el valor del indicador se encuentra entre la media más una desviación estándar; alto, entre la media más dos desviaciones estándar; y muy alto, si los valores se encuentran por arriba de más de dos desviaciones estándar. Por otro lado, el AGEB presenta un nivel de marginación medio-bajo si su valor se encuentra entre la media y menos una desviación estándar; bajo si se encuentra entre la media y menos dos desviaciones estándar; y muy bajo si se encuentra entre la media y menos tres desviaciones estándar.

Del mismo modo, como parte del proceso de obtención del IMU se obtiene una matriz de componentes principales, con el propósito de establecer las ponderaciones asignadas a cada indicador a través del Paquete Estadístico para las Ciencias Sociales (SPSS por sus siglas en inglés).

El valor del índice se determina mediante la siguiente ecuación, con el valor de la media, desviación estándar y sumatoria ponderada de cada uno de los indicadores considerados (CONAPO, 2004):

$$
I M=\sum_{j=1}^{6} a_{j} Z_{i j}(1)
$$

donde:

IM = índice de Marginación Urbana por AGEB

$\mathrm{j}$ = indica cada uno de los indicadores de marginación $(\mathrm{j}=1, \ldots 6)$.

$a_{j}=$ ponderación asignada a cada indicador j de marginación.

$Z_{i j}=$ valor estandarizado de cada indicador $\mathrm{j}$ de marginación, cuyo valor porcentual se resta del promedio y la diferencia es dividida por la desviación estándar de cada indicador.

Posteriormente, se genera la comparativa entre IMU en situación ex ante y ex post y se utiliza el sig para representar los resultados.

\section{Asociación entre condiciones de accesibilidad territorial, IMU e intervenciones técnicas en la red carretera}

La hipótesis que se plantea establece que la accesibilidad carretera de una localidad incide en la calidad de vida de sus habitantes. En este sentido, las localidades con mayores niveles de IMU deben ser las que conectan con carreteras que presentan deficiencias desde el punto de vista de la calidad de su superficie de rodamiento o de sus características geométricas. 
Para determinar lo anterior se asocian tres variables: 1) nivel de accesibilidad relativa y absoluta, 2) condiciones de IMU de las localidades urbanas y suburbanas, y 3) caracterización de las intervenciones técnicas efectuadas en la red carretera de la entidad durante el periodo de análisis.

El ejercicio de asociación se realiza a través de un estudio comparativo por localidad y mediante la superposición de capas de información de las tres variables en un sIG. El nivel de accesibilidad se integra al SIG en dos capas de información: relativa y absoluta. Del mismo modo, la variable IMU se incorpora en situación ex ante (año 2000) y ex post (año 2010), para valorar su evolución. Mientras tanto, las intervenciones se representan organizadas en cada uno de los ańos del periodo de estudio, para determinar los tramos carreteros que han sido atendidos en materia conservación.

Primeramente, se analiza la evolución del imu del año 2000 al 2010, para detectar aquellas localidades que presentan modificaciones en el mismo. Posteriormente, se contrastan las modificaciones con su situación de accesibilidad relativa y absoluta en el año 2010. Por último, en aquellas localidades identificadas, se analiza el nivel de intervención en la red carretera que las conecta durante el periodo de estudio, para verificar si está relacionado con los cambios en los niveles de IMU y la accesibilidad.

\section{Resultados del análisis}

\section{Situación de accesibilidad relativa y absoluta en Baja California}

La ubicación geográfica de las localidades urbanas y suburbanas de Baja California ha definido diferentes comportamientos de accesibilidad relativa; entre dichas localidades, 32 se encuentran en nivel A; 15 en nivel B; y 14 en nivel C. En el municipio de Playas de Rosarito hay dos localidades en nivel A y una en C. Mexicali presenta seis localidades en nivel A, once en $\mathrm{B}$ y dos en $\mathrm{C}$. Tijuana exhibe cinco localidades con nivel A, dos en B y nueve en C. Tecate presenta tres localidades con nivel A y una en B. Mientras tanto, Ensenada muestra 16 localidades con nivel A, dos en B y una en $\mathrm{C}$ (tabla 2).

El comportamiento de cada uno de los municipios es variable en términos de accesibilidad absoluta (tabla 3). Playas de Rosarito y Tecate son las que mantienen una media mayor de carreteras primarias por localidad, de 2 y 1,67, respectivamente; caso contrario es el de las localidades de Ensenada, con una media de 1,13. Por su parte, Tijuana y Mexicali presentan la misma media, con 1,16. Las características de la red primaria varían en la entidad con anchos de $6 \mathrm{~m}$ hasta $20 \mathrm{~m}$, velocidades de $60 \mathrm{~km} / \mathrm{h}$ a $110 \mathrm{~km} / \mathrm{h}$, con pavimentos asfálticos y de terracerías.

Mexicali es el municipio que presenta la media mayor de carreteras alimentadoras por localidad, con 1,63; cabe mencionar que Ensenada y Playas de Rosarito son las que tienen la media menor, con 0,33 y 0 , respectivamente; y Tijuana y Tecate presentan una media de 1 . En la entidad, las características técnicas de las alimentadoras varían con anchos desde $6 \mathrm{~m}$ hasta $12 \mathrm{~m}$, velocidades de $60 \mathrm{~km} / \mathrm{h}$ a $100 \mathrm{~km} / \mathrm{h}$ con pavimentos asfálticos y de concreto hidráulico.

Tijuana, Mexicali y Playas de Rosarito son los municipios que presentan mejor conectividad por caminos secundarios; caso contrario es el de Tecate y Ensenada. Las 
características de estos caminos en la entidad varían con anchos de $6 \mathrm{~m}$ hasta $8 \mathrm{~m}$, velocidades de $60 \mathrm{~km} / \mathrm{h}$ a $80 \mathrm{~km} / \mathrm{h}$ con pavimentos asfálticos y caminos de terracerías.

TABLA 2 Condiciones de accesibilidad relativa de las localidades de Baja California

\begin{tabular}{|c|c|c|c|c|c|}
\hline MUNICIPIO & LOCALIDAD URBANA & $\begin{array}{c}\text { NIVEL } \\
\text { RELATIVO }\end{array}$ & $\begin{array}{c}\text { DISTANCIA } \\
(\mathrm{KM})\end{array}$ & $\begin{array}{c}\text { POBLACIÓN } \\
\text { (HAB.) }\end{array}$ & $\begin{array}{l}\text { CARRETERA } \\
\text { COLINDANTE }\end{array}$ \\
\hline \multirow{3}{*}{ Rosarito } & Playas de Rosarito (Cabecera) & \multirow{2}{*}{ A } & & 65.278 & \multirow{3}{*}{ N.o 1} \\
\hline & Primo Tapia & & & 4.921 & \\
\hline & Ampliación Ej. Plan Libertador & $\mathrm{C}$ & 1,2 & 5.906 & \\
\hline \multirow{19}{*}{ Mexicali } & Mexicali (Cabecera) & \multirow{6}{*}{ A } & \multirow{6}{*}{-} & 689.775 & N.o $2 / \mathrm{N} .{ }^{\circ} 5$ \\
\hline & El Progreso & & & 12.557 & \multirow{4}{*}{ N.o 2} \\
\hline & Puebla & & & 15.168 & \\
\hline & Ejido Sinaloa & & & 1.595 & \\
\hline & Ejido Hechicera & & & 2.517 & \\
\hline & San Felipe & & & 16.702 & N.o 5 \\
\hline & Nuevo León & \multirow{11}{*}{$\mathrm{B}$} & 16,24 & 3.655 & \multirow{9}{*}{ N.o 2} \\
\hline & Guadalupe Victoria & & 31,44 & 17.119 & \\
\hline & Benito Juárez (Ejido Tecolote) & & 2,27 & 4.167 & \\
\hline & Poblado Paredones & & 9,4 & 3.332 & \\
\hline & Ej. Hermosillo & & 0,8 & 5.101 & \\
\hline & \begin{tabular}{|l|} 
Ciudad Morelos (Cuervos) \\
\end{tabular} & & 16,5 & 8.243 & \\
\hline & Vicente Guerrero (Algodones) & & 27,3 & 5.474 & \\
\hline & \begin{tabular}{|l|} 
Santa Isabel \\
\end{tabular} & & 5,0 & 29.311 & \\
\hline & Estación Delta & & 24,21 & 5.180 & \\
\hline & Venustiano Carranza & & 16 & 6.098 & \multirow{2}{*}{ N.o 5} \\
\hline & \begin{tabular}{|l} 
Ciudad Coahuila \\
\end{tabular} & & 33,5 & 5.617 & \\
\hline & Viñas del Sol & \multirow{2}{*}{$\mathrm{C}$} & 3,9 & 2.509 & N.o 2 \\
\hline & Michoacán de Ocampo & & 10,15 & 3.086 & N.o 5 \\
\hline \multirow{16}{*}{ Tijuana } & Tijuana (Cabecera) & \multirow{5}{*}{ A } & \multirow{5}{*}{-} & 1.300 .983 & N.o $1 /$ N.o 2 \\
\hline & La Joya & & & 26.860 & \multirow{2}{*}{ N. ${ }^{\circ} 1$} \\
\hline & Pórticos de San Antonio & & & 34.234 & \\
\hline & El Refugio & & & 36.400 & \multirow{2}{*}{ N.o 2} \\
\hline & Maclovio Rojas & & & 7.279 & \\
\hline & Villa del Campo & $\mathrm{B}$ & 2,42 & 13.906 & N.o 2 \\
\hline & Quinta del Cedro & \multirow{10}{*}{ C } & 4,35 & 5.704 & \multirow{2}{*}{ N.o 1} \\
\hline & \begin{tabular}{|l|} 
Villa del Prado \\
\end{tabular} & & 8,26 & 12.303 & \\
\hline & Terrazas del Valle & & 2,97 & 20.421 & \multirow{8}{*}{ N.o 2} \\
\hline & Las Delicias & & 13,1 & 15.486 & \\
\hline & San Luis & & 6,5 & 8.571 & \\
\hline & Parajes del Valle & & 3,63 & 3.595 & \\
\hline & El Niño & & 3,82 & 8.999 & \\
\hline & Lomas del Valle & & 1,72 & 3.352 & \\
\hline & \begin{tabular}{|l|} 
Los Valles \\
\end{tabular} & & $16,42 / 14,3$ & 3.135 & \\
\hline & Villa del Prado 2a Sección & & 8,2 & 18.226 & \\
\hline
\end{tabular}

(continúa) 
(continuación)

\begin{tabular}{|c|c|c|c|c|c|}
\hline MUNICIPIO & LOCALIDAD URBANA & $\begin{array}{c}\text { NIVEL } \\
\text { RELATIVO }\end{array}$ & $\begin{array}{l}\text { DISTANCIA } \\
\text { (KM) }\end{array}$ & $\begin{array}{l}\text { POBLACIÓN } \\
\text { (HAB.) }\end{array}$ & $\begin{array}{l}\text { CARRETERA } \\
\text { COLINDANTE }\end{array}$ \\
\hline \multirow{4}{*}{ Tecate } & Tecate (Cabecera) & \multirow{3}{*}{ A } & \multirow{3}{*}{--} & 64,764 & No. $2 /$ No. 3 \\
\hline & $\begin{array}{l}\text { Luis Echeverría Álvarez (El } \\
\text { Hongo) }\end{array}$ & & & 2,411 & No. 2 \\
\hline & Nueva Colonia Hindú & & & 4,431 & \multirow{2}{*}{ No. 3} \\
\hline & Lomas de Santa Anita & $\mathrm{B}$ & 2,26 & 6,604 & \\
\hline \multirow{19}{*}{ Ensenada } & Ensenada (Cabecera) & \multirow{16}{*}{ A } & \multirow{16}{*}{--} & 279,765 & \multirow{2}{*}{ No. $1 /$ No. 3} \\
\hline & El Sauzal de Rodríguez & & & 8,832 & \\
\hline & $\begin{array}{l}\text { Rodolfo Sánchez Taboada } \\
\text { (Maneadero) }\end{array}$ & & & 22,957 & \multirow{12}{*}{ No.1 } \\
\hline & Benito García (El Zorrillo) & & & 6,598 & \\
\hline & San Vicente & & & 4,362 & \\
\hline & Ej. Papalote & & & 3,413 & \\
\hline & Ejido México (Punta Colonet) & & & 3,278 & \\
\hline & Camalú & & & 8,621 & \\
\hline & Emiliano Zapata & & & 5,756 & \\
\hline & Vicente Guerrero & & & 11,455 & \\
\hline & Santa Fe & & & 2,632 & \\
\hline & San Quintín & & & 4,777 & \\
\hline & Lázaro Cárdenas & & & 16,294 & \\
\hline & Colonia Nueva Era & & & 3,256 & \\
\hline & $\begin{array}{l}\text { Lázaro Cárdenas (Valle de la } \\
\text { Trinidad) }\end{array}$ & & & 3,366 & \multirow{4}{*}{ No. 3} \\
\hline & Francisco Zarco & & & 2,664 & \\
\hline & $\begin{array}{l}\text { Real del Castillo Nuevo (Ojos } \\
\text { Negros) }\end{array}$ & B & 1,7 & 3,533 & \\
\hline & Rancho Verde & & 6,8 & 2,578 & \\
\hline & $\begin{array}{l}\text { Colonia Lomas de San Ramón } \\
\text { (Triquis) }\end{array}$ & $\mathrm{C}$ & 1,5 & 3,805 & No.1 \\
\hline
\end{tabular}

FUENTE ELABORACIÓN PROPIA 
TABLA 3 Condiciones de accesibilidad absoluta en Baja California en nivel $\mathrm{A}, \mathrm{B}$ y $\mathrm{C}$

\begin{tabular}{|c|c|c|c|c|c|c|}
\hline \multicolumn{2}{|c|}{$\begin{array}{c}\text { NIVEL/ } \\
\text { TIPO DE } \\
\text { CARRETERA }\end{array}$} & MEXICALI & ENSENADA & ROSARITO & TIJUANA & TECATE \\
\hline \multirow{13}{*}{$\begin{array}{l}\text { Nive } \\
\text { A }\end{array}$} & \multirow{4}{*}{ Primarias } & Media: 1,16 & Media: 1,13 & Media: 2 & Media: 1,16 & Media: 1,67 \\
\hline & & \begin{tabular}{|l|} 
Anchos: \\
12 a $20 \mathrm{~m}$ \\
\end{tabular} & $\begin{array}{l}\text { Anchos: } \\
6 \text { a } 20 \mathrm{~m}\end{array}$ & $\begin{array}{l}\text { Anchos: } 12 \mathrm{a} \\
14 \mathrm{~m}\end{array}$ & Anchos: 6 a $12 \mathrm{~m}$ & $\begin{array}{l}\text { Anchos: } \\
8 \text { a } 12 \mathrm{~m}\end{array}$ \\
\hline & & $\begin{array}{l}\text { Velocidades: } 100 \\
\text { a } 110 \mathrm{~km} / \mathrm{h}\end{array}$ & $\begin{array}{l}\text { Velocidades: } \\
60 \mathrm{~km} / \mathrm{h}\end{array}$ & $\begin{array}{l}\text { Velocidades: } \\
100 \text { a } 110 \mathrm{~km} / \mathrm{h}\end{array}$ & $\begin{array}{l}\text { Velocidades: } 100 \\
\text { a } 110 \mathrm{~km} / \mathrm{h}\end{array}$ & $\begin{array}{l}\text { Velocidades: } \\
100 \text { a } 110 \\
\mathrm{~km} / \mathrm{h}\end{array}$ \\
\hline & & $\begin{array}{l}57,14 \% \text { asfalto y } \\
42,86 \% \\
\text { terracerías }\end{array}$ & \multicolumn{4}{|l|}{ Asfalto } \\
\hline & \multirow{2}{*}{$\begin{array}{l}\text { Alimen- } \\
\text { tadoras }\end{array}$} & Media: 1,33 & Media: 0,13 & & Media: 0,44 & \multirow{2}{*}{-- } \\
\hline & & Anchos: 6 a $7 \mathrm{~m}$ & Anchos: $6 \mathrm{~m}$ & & Anchos: $12 \mathrm{~m}$ & \\
\hline & \multirow{2}{*}{$\begin{array}{l}\text { Alimen- } \\
\text { tadoras }\end{array}$} & $\begin{array}{l}\text { Velocidades: } \\
60 \text { a } 90 \mathrm{~km} / \mathrm{h}\end{array}$ & $\begin{array}{l}\text { Velocidades: } \\
60 \mathrm{~km} / \mathrm{h}\end{array}$ & & $\begin{array}{l}\text { Velocidades } 100 \\
\mathrm{~km} / \mathrm{h}\end{array}$ & \multirow{2}{*}{-- } \\
\hline & & \multicolumn{2}{|l|}{ Asfalto } & & $\begin{array}{l}\text { Concreto } \\
\text { hidráulico }\end{array}$ & \\
\hline & \multirow{4}{*}{$\begin{array}{l}\text { Secunda- } \\
\text { rias }\end{array}$} & Media: 2,6 & Media: 0,56 & Media: 2,5 & Media: 2,4 & Media: 0,33 \\
\hline & & Anchos: $6 \mathrm{~m}$ & Anchos: $7 \mathrm{~m}$ & Anchos: $6 \mathrm{~m}$ & Anchos: 6 a $8 \mathrm{~m}$ & - \\
\hline & & \begin{tabular}{|l|} 
Velocidades \\
$70 \mathrm{~km} / \mathrm{h}$ \\
\end{tabular} & \multicolumn{2}{|c|}{ Velocidades $60 \mathrm{~km} / \mathrm{h}$} & $\begin{array}{l}\text { Velocidades: } \\
60 \text { a } 80 \mathrm{~km} / \mathrm{h}\end{array}$ & -- \\
\hline & & $\begin{array}{l}18,75 \% \text { asfalto y } \\
81,25 \% \\
\text { terracerías }\end{array}$ & \begin{tabular}{|l|}
$11,11 \%$ asfal- \\
to y $88,88 \%$ \\
terracerías
\end{tabular} & $\begin{array}{l}60 \% \text { asfalto y } \\
40 \% \text { terracerías }\end{array}$ & $\begin{array}{l}25 \% \text { asfalto y } \\
75 \% \text { terracerías }\end{array}$ & Terracerías \\
\hline & $\begin{array}{l}\text { Localida- } \\
\text { des }\end{array}$ & 6 & 16 & 2 & 5 & 3 \\
\hline \multirow{9}{*}{$\begin{array}{l}\text { Nivel } \\
\text { B }\end{array}$} & \multirow{4}{*}{$\begin{array}{l}\text { Alimen- } \\
\text { tadoras }\end{array}$} & Media: 1,63 & Media: 0,33 & & \multicolumn{2}{|l|}{ Media: 1} \\
\hline & & Anchos: 6 a $7 \mathrm{~m}$ & Anchos: $6 \mathrm{~m}$ & & Anchos: $6 \mathrm{~m}$ & Anchos: $12 \mathrm{~m}$ \\
\hline & & $\begin{array}{l}\text { Velocidades: } \\
70 \text { a } 80 \mathrm{~km} / \mathrm{h}\end{array}$ & $\begin{array}{l}\text { Velocidades: } \\
60 \mathrm{~km} / \mathrm{h}\end{array}$ & & \multicolumn{2}{|c|}{ Velocidades: $60 \mathrm{~km} / \mathrm{h}$} \\
\hline & & \multicolumn{2}{|l|}{ Asfalto } & & \multicolumn{2}{|l|}{ Asfalto } \\
\hline & \multirow{4}{*}{$\begin{array}{l}\text { Secunda- } \\
\text { rias }\end{array}$} & Media: 1,18 & Media: 1 & & & \\
\hline & & Anchos: 6 a $8 \mathrm{~m}$ & Anchos: $7 \mathrm{~m}$ & & & \\
\hline & & $\begin{array}{l}\text { Velocidades: } \\
60 \text { a } 80 \mathrm{~km} / \mathrm{h} \\
\end{array}$ & $\begin{array}{l}\text { Velocidades: } \\
60 \mathrm{~km} / \mathrm{h}\end{array}$ & & & \\
\hline & & $\begin{array}{l}57,15 \% \text { asfalto y } \\
42,85 \% \\
\text { terracerías } \\
\end{array}$ & Asfalto & & & \\
\hline & \begin{tabular}{|l|}
$\begin{array}{l}\text { Localida- } \\
\text { des }\end{array}$ \\
\end{tabular} & 11 & 2 & & 1 & \\
\hline \multirow{5}{*}{$\begin{array}{l}\text { Nivel } \\
\mathrm{C}\end{array}$} & \multirow{4}{*}{$\begin{array}{l}\text { Secunda- } \\
\text { rias }\end{array}$} & \multicolumn{2}{|l|}{ Media: 1} & Media: 2 & \multicolumn{2}{|l|}{ Media: 2,22 } \\
\hline & & Anchos: $7 \mathrm{~m}$ & & $\begin{array}{l}\text { Anchos: } \\
6 \text { a } 12 \mathrm{~m}\end{array}$ & Anchos: 6 a $12 \mathrm{~m}$ & \\
\hline & & $\begin{array}{l}\text { Velocidades: } 80 \\
\mathrm{~km} / \mathrm{h}\end{array}$ & -- & $\begin{array}{l}\text { Velocidades: } \\
60 \mathrm{~km} / \mathrm{h} \\
\end{array}$ & Velocidades: $60 \mathrm{kr}$ & $\mathrm{m} / \mathrm{h}$ \\
\hline & & $\begin{array}{l}50 \% \text { asfalto y } \\
50 \% \text { terracerías }\end{array}$ & Terracerías & Asfalto & $\begin{array}{l}81,81 \% \text { asfalto y } \\
18,19 \% \text { terracería }\end{array}$ & \\
\hline & $\begin{array}{l}\text { Localida- } \\
\text { des }\end{array}$ & 2 & & 1 & 10 & \\
\hline
\end{tabular}

FUENTE ELABORACIÓN PROPIA 


\section{Situación ex ante y ex post de IMU en Baja California}

En el año 2000 hubo un total de 954 AGEB en la entidad, repartiéndose porcentualmente en $10 \%$ en situación baja de IMU, $50 \%$ en situación media baja, $24 \%$ en situación media alta, $12 \%$ en situación alta y $4 \%$ en situación muy alta. En 2010 aumentó a 1,515 AGEB; a pesar del incremento de 561 AGEB (58\%), se sigue manteniendo el IMU en situación muy alta, con el 4\%; la situación en alta mostró un descenso del $7 \%$, la media alta bajó únicamente $1 \%$, la media baja aumentó un $18 \%$ y la baja desapareció.

El municipio de Tijuana pasó de 388 ageb en el año 2000 a 571 Ageb en el 2010, incrementando 183 AGEB. La cantidad de AGEB en situación media baja tuvo un incremento de $60 \%$ a $68 \%$, lo que denota la desaparición de la condición baja (figura 3). Asimismo, disminuye la situación alta de $8 \%$ a $4 \%$; y la muy alta, de $3 \%$ a $1 \%$. Cabe mencionar que las 16 localidades consideradas mejoraron sus condiciones de IMU de un año a otro.

FIgUra 3 | Situación de IMU de ageb en el municipio de Tijuana

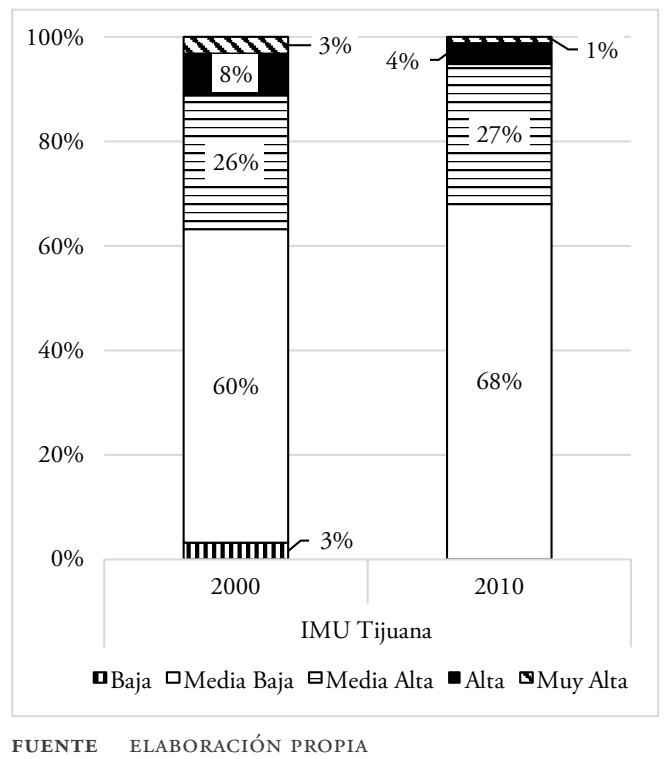

En 2000, el municipio de Mexicali contaba con 289 AGEB, mientras que en 2010 aumentó a 468 AGEB, incrementando 179 AGEB. La concentración de AGEB en el año 2000 fue de 23\%, misma que desapareció en el 2010 (figura 4). Las situaciones de alta y media baja se redujeron; sin embargo, no fueron tan notorias. Caso contrario, la media baja tuvo un incremento importante, pasando de $47 \%$ a $78 \%$. La mayoría de las localidades mejoró sus condiciones de IMU, ya que de las 19 localidades, solo dos suburbanas empeoraron sus condiciones de marginación.

El municipio de Ensenada pasó de 187 AGEB a 343 AgEb de un año a otro, lo que indica un crecimiento de 156 AGEB. La situación del IMU en media baja aumentó de 
$40 \%$ a $57 \%$, desapareciendo la condición baja. Además, disminuyó el porcentaje en alta de $17 \%$ a $10 \%$ (figura 5). Asimismo, se incrementó la situación muy alta, de $7 \%$ a 14\%. Cinco localidades suburbanas empeoraron sus condiciones de IMU. A pesar de aparecer AGEB en situación media baja y media alta en seis localidades suburbanas, aumentaron la cantidad de AGEB en situación alta y muy alta.

FIgURA 4 Situación de ImU de AgEB en el municipio de Mexicali

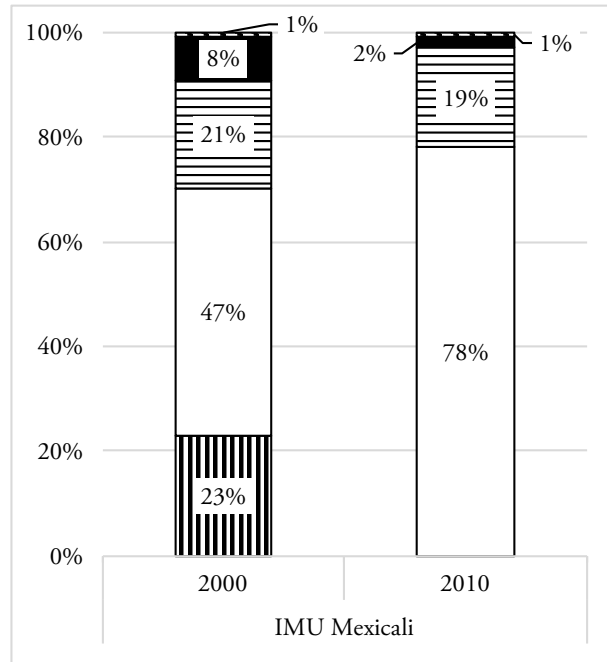

$\mathbf{0}$ Baja $\square$ Media Baja $\boxminus$ Media Alta Alta $\mathbf{\square}$ Muy Alta

FUENTE ELABORACIÓN PROPIA

FIgURA 5 Situación de ImU de AGEB en el municipio de Ensenada

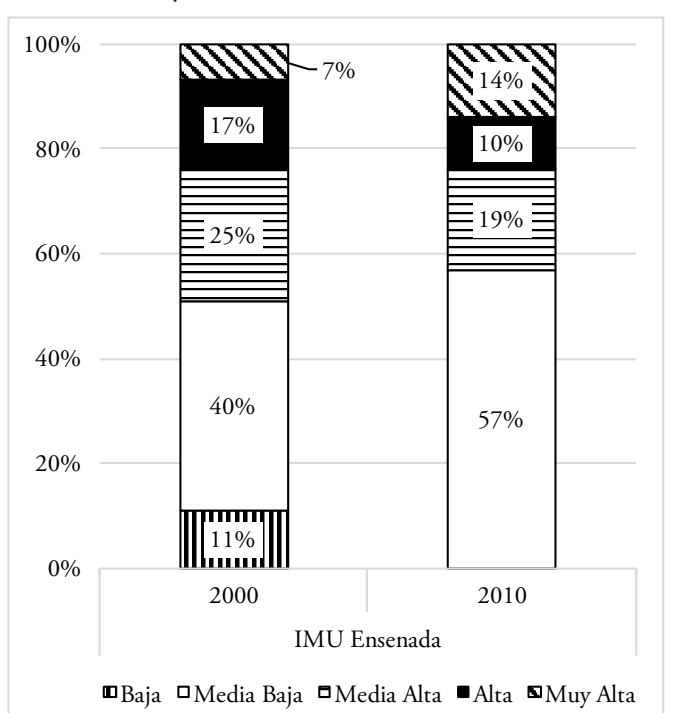

FUENTE ELABORACIÓN PROPIA 
El municipio de Playas de Rosarito registró 53 AgEb en el año 2000 y 68 AgEb en 2010, incrementando 15 AGEB. En el 2000 concentró un 76\% del IMU, al sumar la situación media alta y alta (figura 6). Dicho municipio no presentó AGEB en situación baja en ambos años. En 2010, disminuyó el IMU en situación alta y desapareció la condición muy alta. La mayoría de las AGEB aparecen en situación media baja, con $65 \%$.

Figura 6 | Situación de Imu de Ageb en el municipio de Playas de Rosarito

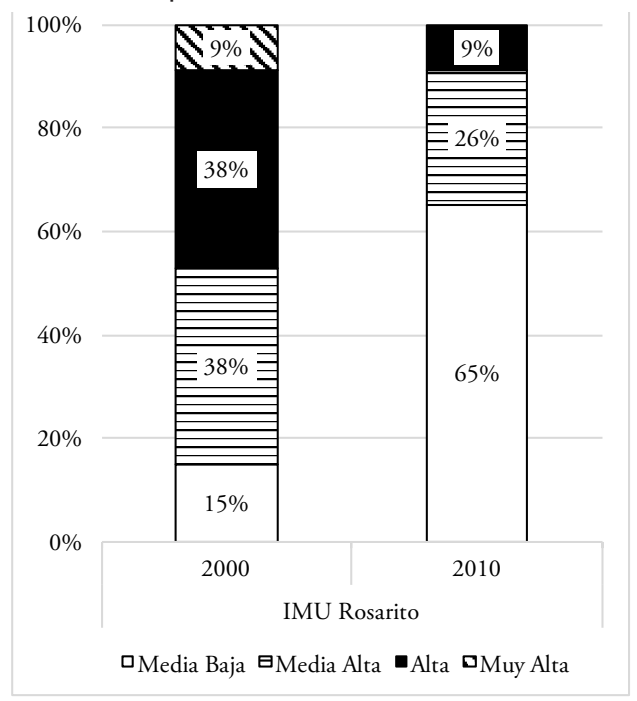

FUENTE ELABORACIÓN PROPIA

FIgURA 7 Situación de Imu de AGEB en el municipio de Tecate

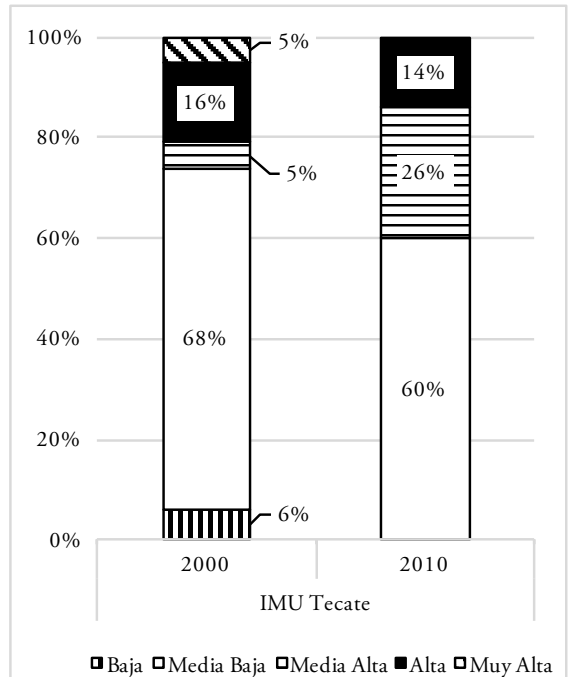

FUENTE ELABORACIÓN PROPIA 
En el 2000, el municipio de Tecate contaba con 37 AGEB y en 2010 registró 65, incrementando 28 AGEB. Como el resto de municipios, el IMU en situación baja se redujo a 0\% (figura 7). En 2010, la mayoría de las AGEB se concentraron en la situación media baja, siguiendo en media alta y en alta.

\section{Intervenciones técnicas en la red carretera federal de Baja California}

La inversión en la red federal ha favorecido principalmente a tramos primarios donde se ha ejecutado algún tipo de intervención relacionado a conservación. Cabe mencionar que algunos tramos no se han intervenido desde el año 2000.

En 2000 se trabajaron 143,5 kilómetros en la red federal primaria, distribuidos en tres municipios y representando el 6,5\% del total del periodo de estudio. En Mexicali, se intervino en la N. ${ }^{\circ} 5$ en San Felipe y en la N.o 2. En Ensenada, en la N. ${ }^{\circ}$ 3, en la N. ${ }^{\circ}$ cerca de la cabecera municipal y en otro tramo ubicado al sur, lejos de las localidades suburbanas. En Tecate, en la N. ${ }^{\circ} 2$, colindando con Mexicali, y en la N. ${ }^{\circ} 3$ en los límites con Ensenada.

En 2001, fueron 160 kilómetros los trabajados en la red federal primaria, distribuidos en cuatro municipios y representando el 7,2\% del total del periodo de estudio. En Mexicali, en la N.o 2. En Ensenada, en la N.o 3 interconectando con Mexicali y en diferentes partes de la N. ${ }^{\circ} 1$. En Tijuana, en la N. ${ }^{\circ} 2$ cerca de Tecate y Playas de Rosarito. En Tecate, en la N. ${ }^{\circ} 2$ y en la N.o 3 al sur de la cabecera.

En 2002, fueron 270,4 kilómetros en la red federal primaria, distribuidos en tres municipios y representando el 12,1\% del total del periodo de estudio. En Mexicali, en la N. ${ }^{\circ} 2$, comunicando con Tecate y también en la N. ${ }^{\circ}$. En Tecate, en la N. ${ }^{\circ}$ 2 y N.o 3. En Ensenada, en la N.o 3 en Francisco Zarco y en la N.o 1 al sur de la cabecera municipal.

En 2003, fueron 172,3 kilómetros distribuidos en la red federal primaria y alimentadora, en cuatro municipios y representando el 7,8\% del total del periodo de estudio. En Mexicali, en la N.o 2, cercano a los límites con Tecate, y en la N.o 5. En Tecate, en la N. ${ }^{\circ}$ 2, colindando con Mexicali. En Ensenada, en la N.o 3, conectando con Mexicali, y en la N. 1 al sur de la cabecera, en el Ejido Papalote y en un tramo alimentador. En Tijuana, en un tramo alimentador localizado al sur de la cabecera municipal.

En 2004, fueron 214,2 kilómetros distribuidos en la red federal primaria y alimentadora, en tres municipios y representando el 9,7\% del total en el periodo de estudio. En Mexicali se intervino en la N. ${ }^{\circ}$ 2, cercano a Tecate, y en la N.o 5 , próximo a San Felipe. En Tecate, en la N. ${ }^{\circ} 2$, cercano a Mexicali, y también en la N. ${ }^{\circ} 3$. En Ensenada, en la N. ${ }^{\circ} 3$ colindando con Tecate; y en diferentes tramos de la N. ${ }^{\circ}$, uno al norte de la cabecera, otro al sur de Ejido Papalote y en dos tramos alimentadores.

En 2005, fueron 250 kilómetros distribuidos en la red federal primaria y alimentadora, en cuatro municipios y representando el $11,3 \%$ del total del periodo de estudio. En Mexicali se trabajó en la N. ${ }^{\circ}$ 2, cerca de la cabecera municipal, en la N. ${ }^{\circ} 5$ en San Felipe y en un tramo alimentador. En Tecate, en la N. ${ }^{\circ} 2$ colindando con Mexicali y en la localidad de El Hongo; también en la N. 3 cerca de Ensenada. En Playas de Rosarito, en la N.o 1 al sur del municipio. En Ensenada, en la N. ${ }^{\circ} 1$ 
en El Sauzal, en la N. ${ }^{\circ} 5$ en San Felipe y en un tramo alimentador que interconecta con la N.o 3.

En 2006, fueron 152,7 kilómetros distribuidos en la red federal primaria, en tres municipios y representando el 6,8\% del total del periodo de estudio. En Mexicali, en la N. ${ }^{\circ} 2$ y en la N. ${ }^{\circ} 5$ en San Felipe. En Tijuana, en la N. ${ }^{\circ} 2$ cerca de Tecate. En Ensenada, en la N. ${ }^{\circ} 1$ y en la N.o 5.

En 2007, fueron 183,4 kilómetros en la red federal primaria y alimentadora, en cuatro municipios y representando el 8,3\% del total del periodo de estudio. En Mexicali, en la N. ${ }^{\circ} 2$ cerca del estado de Sonora y en El Progreso; también en la N. ${ }^{\circ}$, dando acceso a varias localidades suburbanas, y en un tramo alimentador. En Tecate, en la N.o 2 en El Hongo y en otro tramo próximo a Tijuana. En Ensenada, en la N. 3 en Francisco Zarco y Real del Castillo y en la N. ${ }^{\circ} 1$ cerca de Playas de Rosarito. En Tijuana, en la N.o 2 y en la N.o 1.

En 2008, fueron 199,7 kilómetros en la red federal primaria y alimentadora, en cuatro municipios y representando el $9 \%$ del total del periodo de estudio. En Mexicali, en la N.o 2 cerca de Sonora y en El Progreso; también en la N.o 5 en San Felipe y en un tramo alimentador. En Tecate, en la N.o 2 colindando con Mexicali y en la N. 3 en la Nueva Colonia Hindú. En Tijuana, en la N. ${ }^{\circ}$ 2. En Ensenada, en la N.o 3 en Francisco Zarco, en la N. ${ }^{\circ}$, en la N. ${ }^{\circ} 1$ y en San Vicente.

En 2009, fueron 267,5 kilómetros en la red federal primaria y alimentadora, en los cinco municipios y representando el $12 \%$ del total del periodo de estudio. En Mexicali, en la N. ${ }^{\circ} 2$ cerca de Tecate, en la N. ${ }^{\circ} 5$ próximo a la cabecera municipal, en San Felipe y en un tramo alimentador. En Tecate, en la N.o 2 en El Hongo, en la N.o 3 en la Nueva Colonia Hindú y en los límites con Ensenada. En Tijuana, en la N. ${ }^{\circ} 1$. En Ensenada, en la N. 3 en Francisco Zarco, El Sauzal y Valle de la Trinidad, en la N. ${ }^{\circ} 1$ en Benito García y en la N. ${ }^{\circ}$ 5. En Playas de Rosarito, en la N. ${ }^{\circ} 1$ en Primo Tapia.

Finalmente, en 2010 fueron 209,2 kilómetros distribuidos en la red federal primaria y alimentadora, en cuatro municipios y representando el 9,4\% del total del periodo de estudio. En Mexicali, en la N. ${ }^{\circ} 2$ cerca de Tecate y en la N. ${ }^{\circ} 5$. En Tecate, en la N.o 2 y N. 3 en Nueva Colonia Hindú. En Tijuana, en la N.o 2. En Ensenada, en la N.o 3 en Real del Castillo y Valle de la Trinidad, en la N. ${ }^{\circ} 1$ y en tramos alimentadores.

\section{Resultados de asociación e identificación de situaciones críticas}

En el municipio de Ensenada, cinco de sus localidades suburbanas presentan IMU en situación desfavorable: Colonia Lomas de San Ramón, Emiliano Zapata, Camalú, Benito García y Rodolfo Sánchez Taboada. Las últimas dos, aunque se encuentran cerca de la cabecera municipal, no registraron en su red carretera intervenciones con trabajos de conservación durante el periodo de estudio. A la última localidad la intercepta un tramo alimentador y se conecta a la red a través de una carretera primaria con un ancho de $8 \mathrm{~m}$, permitiendo velocidades de $60 \mathrm{~km} / \mathrm{h}$ sobre una superficie asfaltada. Emiliano Zapata y Camalú son localidades vecinas, se encuentran en la misma condición de accesibilidad relativa, pero más alejadas de la cabecera municipal. El tramo de red que las interconecta no se interviene desde 2006 y tiene un ancho de $6 \mathrm{~m}$, velocidad de operación de $60 \mathrm{~km} / \mathrm{h}$ y cuenta con superficie de asfalto. 
Por su parte, Vicente Guerrero presenta un IMU más favorable, y el tramo primario que la intercepta presenta un ancho de sección de $12 \mathrm{~m}$. En la Colonia Lomas de San Ramón la situación es más crítica, con un 100\% de AGEB con IMU en condición de muy alta marginación. Se encuentra en un nivel de accesibilidad relativa Nivel C, pues el camino que la intercomunica con la red general es de terracerías, y no ha sido intervenido durante el periodo de estudio.

Las localidades de Mexicali mejoraron la situación de su IMU en lo general, pero la mayor cantidad de AGEB se encontró en una situación muy alta en áreas suburbanas. La mayoría de las localidades se encuentra en un nivel de accesibilidad relativa $\mathrm{B}$, con tramos con anchos de sección que rondan entre los $6 \mathrm{~m}$ y $8 \mathrm{~m}$. Este municipio registra muchas intervenciones en su red primaria durante el periodo de estudio, aunque pocas en la red alimentadora y secundaria.

La situación de Tijuana es similar a Mexicali, ya que sus localidades mejoraron significativamente su condición de marginación del año 2000 al 2010. En diez localidades la accesibilidad relativa dio $\mathrm{C}$, con tramos de red con secciones entre los $6 \mathrm{~m}$ y $12 \mathrm{~m}$. El nivel de intervenciones fue favorable durante el periodo de estudio, sobre todo en la red primaria y, en menor medida, en alimentadoras y secundarias.

El municipio de Tecate presenta una situación particular, ya que sus cuatro localidades exhiben una tendencia de IMU en media baja y media alta, desapareciendo condiciones extremas bajas y muy altas. Las intervenciones que se efectuaron fueron constantes durante el periodo de estudio, principalmente en la red primaria. Lomas de Santa Anita es la única localidad que presenta condiciones de accesibilidad relativa B y la alimentadora que la conecta presenta anchos de sección de $12 \mathrm{~m}$, velocidades de operación de $60 \mathrm{~km} / \mathrm{h}$ y pavimento asfáltico. Las localidades restantes son interceptadas por carreteras primarias con anchos entre $8 \mathrm{~m} \mathrm{y} 12 \mathrm{~m}$, velocidades de operación entre los 60 y $100 \mathrm{~km} / \mathrm{h}$, y también con superficie en asfalto.

En 2010, las tres localidades urbanas y suburbanas que integran el municipio de Playas de Rosarito mejoraron considerablemente su situación de IMU. La cabecera y Primo Tapia interceptan con carreteras primarias con anchos de sección de $12 \mathrm{~m}$ y $14 \mathrm{~m}$, permitiendo velocidades de operación de hasta $110 \mathrm{~km} / \mathrm{h}$ sobre superficies de asfalto. Por otro lado, Ejido Plan Libertador accede a la red carretera a través de ejes secundarios. Las intervenciones técnicas que se efectuaron en la red y que impactan en sus localidades se suscitaron en 2001, 2005, 2007 y 2009.

\section{Conclusiones y recomendaciones}

Este estudio exhibe que la infraestructura carretera es un elemento físico que promueve el desarrollo socioeconómico de áreas marginadas. La accesibilidad que brinda una red carretera construida con condiciones técnicas adecuadas, potencializa el equilibrio territorial y mejora la calidad de vida de las personas a través del acceso a los servicios básicos. Las intervenciones técnicas en materia de conservación sobre las carreteras contribuyen a la mejora de la accesibilidad territorial.

Los niveles críticos de accesibilidad carretera pueden evidenciar ausencia de políticas de desarrollo en áreas con escaso nivel de urbanización, ya que los lugares que los presentan coinciden con aquellos que muestran los niveles más severos en su condición socioeconómica. 
Los niveles del IMU permiten identificar las condiciones de desarrollo social en áreas geográficas específicas, medidas por tipos de vivienda y acceso a servicios de salud y de educación. Se ha encontrado que las zonas que exhiben disminución en los niveles de marginación coinciden espacialmente, en su mayoría, con la presencia de intervenciones que han mejorado el estado de las carreteras durante el periodo de estudio.

En todos los municipios de Baja California se ha priorizado intervenir en carreteras primarias, opción que representa un $88,8 \%$ del total de la inversión en la red federal. Esto, sin duda, ha contribuido de manera favorable a la mejora de las condiciones de la población de muchas localidades con alto peso poblacional dentro de la entidad. Sin embargo, este estudio arroja que las intervenciones en la red alimentadora y secundaria, específicamente en áreas urbanas y suburbanas que presentan escasa conectividad y bajos niveles de calidad de vida, requieren de mayor prioridad. Las localidades suburbanas presentan actualmente niveles de IMU más desfavorables, principalmente aquellas alejadas de la cabecera municipal. Algunas localidades suburbanas conectadas a la red por tramos alimentadores y secundarios tienen, al menos, las mismas condiciones de acceso a servicios básicos que otras localidades urbanas. Un aspecto necesario de considerar es que, actualmente, el $84,7 \%$ de la población de la entidad se encuentra conectado por la red primaria y el $15,3 \%$ restante lo hace a través de ramales alimentadores o vías secundarias.

En general, las localidades de los municipios de Mexicali, Tijuana y Tecate exhiben buenas condiciones de calidad de vida, con una red carretera bien abastecida por carreteras primarias, alimentadoras y secundarias. Sin embargo, requieren mejoras técnicas en la red alimentadora y secundaria, principalmente Tijuana y Tecate, que deben incorporar tramos alimentadores. Por su parte, el municipio de Ensenada es el que presenta la mayor cantidad de AGEB con niveles de IMU en condiciones altas y muy altas, lo que se atribuye a la falta de tramos alimentadores y de mejoras técnicas necesarias en la red primaria existente. Aunque la extensión territorial de este municipio es muy grande, las intervenciones registradas hasta ahora han beneficiado a ciertas localidades que ya presentan niveles bajos de marginación. Asimismo, las localidades del municipio de Playas de Rosarito han mejorado sustancialmente su calidad de vida, porque coincide que casi toda su población está conectada a la red primaria, alimentadora y secundaria. Aun así, prevalece una pequeña cantidad de población que requiere de tramos alimentadores para incorporarse de mejor manera a la red.

En el análisis de accesibilidad absoluta, se concluye que el factor de ancho de sección carretera es un aspecto importante que repercute en los niveles de IMU. Por su parte, la distancia de las localidades con la red primaria no entrega conclusiones claras sobre su incidencia en el comportamiento del IMU. Lo mismo para la velocidad de operación.

Finalmente, se determina que las condiciones de accesibilidad relativa y absoluta que presentan las localidades de Baja California repercuten en los niveles de IMU y, por ende, en la calidad de los servicios de educación, salud y vivienda que recibe su población. 


\section{Referencias bibliográficas}

Aparicio, C., Jaramillo, M. \& San Romàn, C. (2011). Desarrollo de la infraestructura y reducción de la pobreza: el caso peruano. Informe final de proyecto informe final del Proyecto Breve ganador (рв32-201о) en el Concurso de Investigación 2010, organizado de manera conjunta por el Consorcio de Investigación Económica y Social (CIEs) y el Centro Internacional de Investigaciones para el Desarrollo (IDRC). Lima: CIEs, Universidad del Pacífico, Centro de Investigación. En https://bit.ly/2aBmzpE

Bel, G. (2010). La racionalización de las infraestructuras de transporte en España. Cuadernos Económicos de ICE, (80), 211-228. En https://dialnet.unirioja.es/servlet/ articulo? codigo $=3708336$

Bistrain, C. (2010). Revisión de los índices de marginación elaborados por el conapo. Estudios Demográficos y Urbanos, 25(1), 175-217. En http://estudiosdemograficosyurbanos. colmex.mx/index.php/edu/article/view/1371

Bolton, R.. (1990). Reseña de Technology and the rise of the networked city in Europe and America, de J. Tar \& G. Dupuy (eds.). En Technology and Culture, 31(2), 299-301. https://doi. org/10.2307/3105667

Cárdenas, O. (2010). Cardenalización del índice de marginación: una metodología para evaluar la eficiencia del gasto ejercido en el Ramo 33. EconoQuantum, 7(1), 41-66. En http:// www.revistascientificas.udg.mx/index.php/EQ/article/view/119

Chi, G. (2010). The impacts of highway expansion on population change: an integrated spatial approach. Rural Sociology, 75(1), 58-89. https://doi.org/10.1111/j.15490831.2009.00003.x

Chías Becerril. L., Iturbe Posadas, A. \& Reyna Sáenz, F. (2001). Accesibilidad de las localidades del Estado de México a la red carretera pavimentada: un enfoque. Investigaciones Geográficas (Boletín del Instituto de Geografía, unAm), (46), 117-130. En http://www. scielo.org.mx/pdf/igeo/n46/n46a9.pdf

Cipoletta, G., Pérez, G. \& Sánchez, R. (2010). Politicas integradas de infraestructura, transporte y logistica: experiencias itnternacionales y propuestas inciales. Serie Recursos naturales e infraestructura, 150 (LC/L.3226-P). Santiago, Chile: Comisión Económica para América Latina y el Caribe (CEPAL). En https://repositorio.cepal.org/bitstream/ handle/11362/6350/1/S1000312_es.pdf

Consejo Nacional de Población (CONAPo). (2004). Índice absoluto de marginación 1990-2000. México, D.F.: Conapo. En https://bit.ly/2KdU2I1

Consejo Nacional de Población (CONAPo). (2010). Metodología de estimación del índice de marginación urbana 2010. Anexo C. de Metodología para la medición multidimensional de la pobreza en México. México, D.F.: Conapo. En https://bit.ly/2ozlgxQ

Consejo Nacional de Población (CONAPo). (2012). Índice de Marginación Urbana 2010. D.F.: CONAPO. En http://www.conapo.gob.mx/en/CONAPO/Indice_de_marginacion_ urbana_2010

Coronado, J. M. \& Garmendia, M. (2008). Carreteras-planeamiento. Algunas claves de la evolución histórica de una relación imperfecta. Ciudades: Revista del Instituto Universitario de Urbanistica de la Universidad de Valladolid, (11), 33-51. https://doi. org/10.24197/ciudades.11.2008.33-51 
De Rus Mendoza, G. (1996). Infraestructuras, crecimiento regional y evaluación económica. Papeles de Economía Española, (67), 222-237.

Farrow, A. \& Nelson, A. (2001). Modelación de la accesibilidad en ArcView 3: Una extensión para calcular el tiempo de viaje y obtener información sobre captación de mercados. Cali: Centro Internacional de Agricultura Tropical (сіAT). En https://bit.ly/2HrEUJk

Geurs, K. T. \& Van Wee, B. (2004). Accessibility evaluation of land-use and transport strategies: review and research directions. Journal of Transport Geography, 12(2),127-40. https:// doi.org/10.1016/j.jtrangeo.2003.10.005.

Gutiérrez Gallego, J. A., Mora Aliseda, C., Gómez Domínguez, P. \& Jaraíz Cabanillas, F. J. (2010). Accesibilidad de la población a las aglomeraciones urbanas de la Península Ibérica. Finisterra-Revista Portuguesa de Geografia, 14(89), 107-118. En http://www. scielo.mec.pt/pdf/fin/n89/n89a06.pdf

Gutiérrez Puebla, J. \& Monzón de Cáceres, A. (1993). La accesibilidad a los centros de actividad económica antes y después del plan. Estudios Territoriales, (97), 385-395.

Holl, A. (2011). Factors influencing the location of new motorways: large scale motorway building in Spain. Journal of Transport Geography, 19(6), 1282-1293. https://doi. org/10.1016/j.jtrangeo.2011.06.006

Instituto Nacional de Estadística y Geografía (INEGI). (2000). XII Censo general de población $y$ vivienda. Aguascalientes, México. En http://www.beta.inegi.org.mx/proyectos/ ccpv/2000/

Instituto Nacional de Estadística y Geografía (INEGI). (2010a). XIII Censo de población y vivienda. Aguascalientes, México. En http://www.inegi.org.mx/est/contenidos/proyectos/ accesomicrodatos/cpv2010/default.aspx

Instituto Nacional de Estadística y Geografía (INEGI). (2010b). Compendio de criterios y especificaciones técnicas para la generación de datos e información de carácter fundamental. Aguascalientes, México. En http://www.inegi.org.mx/inegi/spc/doc/ INTERNET/16-\%20marco_geoestadistico_nacional.pdf

Instituto Nacional de Estadística y Geografía (INEGI). (2011). Anuario estadístico por entidad federativa 2011. Aguascalientes, México. En https://bit.ly/2r0e6F2

International Road Federation (IRF). (2007). The socio-economic benefits of roads in Europe. Brussels, Belgium: Researh Council (IRF), European Union Road Federation (ERF), International Road Federation (IRF) - Brussels Programme Centre. En https://bit. ly/2qXMukM

López, E. \& Monzón, A. (2008). Metodología para la evaluacion de planes de infraestructura de transportes: un enfoque integral. En Actas del VIII Congreso de Ingeniería de Los Transportes (s/p), La Coruña, Espańa, 2-4 de julio de 2008. En http://oa.upm.es/3206/

Loyola, C. (2005). Infraestructura vial y niveles de accesibilidad entre los centros poblados y los centros de actividad económica en la provincia de Nuble, viII Región. Tiempo y espacio (Universidad del Bío-Bío, Departamento de Ciencias Sociales, Chillán), 12(15), 101-112. En http://studylib.es/doc/8066083/infraestructura-vial-y-nivelesde-accesibilidad-entre-los...

Loyola, C. \& Albornoz, E. (2009). Flujo, movilidad y niveles de accesibilidad en el centro de Chillán. Año 2007. Propuesta de mejoramiento mediante sig. Urbano, 12(19), 17-27. En http://revistas.ubiobio.cl/index.php/RU/article/view/332 
Lucioni, L. (2009). La provisión de infraestructura en América Latina: tendencia inversiones y financiamiento. Serie Macroeconomía del desarrollo, 72 ([E/]LC/L.298 I-P). Santiago, Chile: Comisión Económica para América Latina y el Caribe (CEPAL). En http:// archivo.cepal.org/pdfs/2008/S0800783.pdf

Martínez, H. (2012). La accesibilidad regional y el efecto territorial de las infraestructuras de transporte. Aplicación en Castilla-La Mancha. Boletín de la Asociación de Geógrafos Españoles, (59), 79-103. En http://www.age-geografia.es/ojs/index.php/bage/article/ viewFile/1450/1371

Monzón, A. (1988). Los indicadores de accesibilidad y su papel decisor en las inversiones en infraestructuras de transporte. Aplicaciones en la comunidad de Madrid. Tesis Doctoral. Universidad Politécnica de Madrid. En http://oa.upm.es/736/

Mungaray Moctezuma, A. \& García, L. (2014). La influencia de la carretera Mexicali-San Felipe en la calidad de vida de sus inmediaciones. Estudios Sociales, 23(46), 190-212. En https://dialnet.unirioja.es/servlet/articulo?codigo $=5831980$

Mungaray Moctezuma, A. \& Luque, R. (2013). Carreteras federales en Baja California: 20082012. Mexicali, Baja California: Gobierno del estado de Baja California / Universidad Autónoma de Baja California.

Nogales, J. M., Figueira, J., Gutiérrez, J. A., Pérez, J. A. \& Cortés, T. (2002). Determinación de la accesibilidad a los centros de actividad económica de extremadura mediante técnicas SIG. XIV Congreso Internacional de Ingeniería Gráfica: Santander, 5-7 junio de 2002. En http://81.47.175.201/TRACC/attachments/article/11/2002_Accesibilidad_ extremadura.pdf

Nogués, S. \& Salas, H. (2007). El impacto de las carreteras en el desarrollo urbano. España, Colegio de Ingeniero de Caminos, Canales y Puertos. Biblioteca Digital. También en I Congreso de Urbanismo y Ordenación del Territorio. "Ciudad y Territorio". Bilbao, 7-9 mayo 2008.

Obregón-Biosca, S. A. (2010). Estudio comparativo del impacto en el desarrollo socioeconómico en dos carreteras: Eix transversal de Catalunya, España, y MEXi20, México. Economía, Sociedad y Territorio, 10(32), 1-47. En http://www.scielo.org.mx/pdf/est/v10n32/ v10n32a2.pdf

Rodríguez, J. (2003). La pobreza como marginación y delito. Gazeta de Antropología, 19 (art. 14), 1-39. http://www.ugr.es/ pwlac/G19_14Jose_Rodriguez_Molina.pdf

Romero, M. (2001): Evaluación económica de inversiones en infraestructuras. Revista Valenciana de Economía y Hacienda, (3), 47-66.

Rozas, P. \& Sánchez, R. (2004). Desarrollo de infraestructura y crecimiento económico: una revisión conceptual. Serie Recursos naturales e infraestructura, 75 (LC/L.2182-P). Santiago, Chile: Comisión Económica para América Latina y el Caribe (CEPAL). En https://repositorio.cepal.org/bitstream/handle/11362/6441/1/S048642_es.pdf

Secretaría de Comunicaciones y Transportes (scT). (2008). Consideraciones sociodemográficas complementarias a la evaluación económica de proyectos de infraestructura carretera. Publicación Técnica N. ${ }^{\circ}$ 310. Realizado por J. A. Arroyo Osorno, G. Torres Vargas \& S. Hernández García. Ciudad de México y Sanfandila, Querétaro. En http://www.imt. $\mathrm{mx} /$ archivos/publicaciones/publicaciontecnica/pt310.pdf 
Secretaría de Comunicaciones y Transportes (sCT). (2008). (2009). El sector carretero en México (Opúsculo). Red Nacional de Carreteras. México, D.F.: sct. En http://www.sct.gob. $\mathrm{mx} /$ fileadmin/DireccionesGrales/DGDC/Publicaciones/Opusculo/opusculo.pdf

Secretaría de Comunicaciones y Transportes (scT). (2011). Dictamen de factibilidad técnica, económica y ambiental de la carretera Mexicali-San Felipe. México D.F.: scT.

Taylor, M., Sekhar, S. \& D’Este, G. M. (2006). Application of accessibility based methods for vulnerability analysis of strategic road networks. Networks and Spatial Economics, 6(34), 267-291. https://doi.org/10.1007/s11067-006-9284-9

Varela, F. A. (2013). Análisis geoespacial para la caracterización funcional de las infraestructuras viarias en modelos de accesibilidad territorial utilizando sistemas de información geográfica. Tesis doctoral. Departamento de Métodos Matemáticos y de Representación, Escuela Técnica Superior de Ingeniería de Caminos, Canales y Puertos, A Coruña. En https://core.ac.uk/download/pdf/61909431.pdf

Vassallo, J. M. \& Izquierdo, R. (2010). Infraestructura pública y participación privada: conceptos y experiencias en America y España. Bogotá: Corporación Andina de Fomento. En https://www.caf.com/media/3163/LibroinfraestructuraFINAL.pdf

Zaragoza, A. (1999). Las carreteras: sistema medular de transporte. En M. Aymerich, R. Izquierdo, A. Zaragoza, R. Gistau, J. Nasarre, Á. Fernández-Aller, E. Zurutuza, R. Molina (...), C. de Andrés, Infraestructuras y Crecimiento Económico (pp. 127-198). Madrid: Fundación para el Análisis y los Estudios Sociales (FAEs). 